\title{
Determination of interface properties between micron-thick metal film and ceramic substrate using peel test
}

\author{
Haifeng Zhao • Yueguang Wei
}

Received: 9 August 2006 / Accepted: 27 April 2007 / Published online: 1 June 2007

(C) Springer Science+Business Media B.V. 2007

\begin{abstract}
Peel test measurements have been performed to estimate both the interface toughness and the separation strength between copper thin film and $\mathrm{Al}_{2} \mathrm{O}_{3}$ substrate with film thicknesses ranging between 1 and $15 \mu \mathrm{m}$. An inverse analysis based on the artificial neural network method is adopted to determine the interface parameters. The interface parameters are characterized by the cohesive zone $(\mathrm{CZ})$ model. The results of finite element simulations based on the strain gradient plasticity theory are used to train the artificial neural network. Using both the trained neural network and the experimental measurements for one test result, both the interface toughness and the separation strength are determined. Finally, the finite element predictions adopting the determined interface parameters are performed for the other film thickness cases, and are in agreement with the experimental results.
\end{abstract}

Keywords Interfacial toughness - Metal thin film . Peel test · Cohesive zone model · Inverse analysis

H. Zhao $(\varangle) \cdot$ Y. Wei $(\varangle)$

State-Key Laboratory of Nonlinear Mechanics (LNM), Institute of Mechanics, Chinese Academy of Science, Beijing 100080, P.R. China e-mail: Zhf@LNM.imech.ac.cn

Y. Wei

e-mail: Ywei@LNM.imech.ac.cn

\section{Introduction}

In recent years, with extensive applications of thin film systems in engineering, research on investigating the mechanical behaviors of the interfaces between thin films and substrates has attracted a great deal of attention and interest (Cotterell et al. 2006; Hadavinia et al. 2006; Pardoen et al. 2005; Wei 2002, 2004; Cui etal.; Song and Jin 2002; Yang and Thouless 2001; Yang etal. 1999, 2001; Park and Yu 1998; Park etal. 1999). In the present research we will focus our attention on investigating the mechanical properties of the ultra thin metal film $(\mathrm{Cu})$ on a ceramic substrate $\left(\mathrm{Al}_{2} \mathrm{O}_{3}\right)$ which plays a crucial part in microelectronics packing (Song and Jin 2002; Park and Yu 1998; Park etal. 1999). In the mechanical behavior characterization of elastic film/substrate systems, usually, interfacial adhesion energy per unit area $\Gamma_{0}$ (or fracture toughness) is adopted as an important interface parameter. $\Gamma_{0}$ is typically measured using a peel test method (Hadavinia et al. 2006; Pardoen etal. 2005; Asai et al. 2001; Bundy et al. 2000; Dillard and Pocius 2002; Ferracin et al. 2003). However, for the case of a ductile film (elastic-plastic), besides the interface adhesion energy $\Gamma_{0}$ interfacial separation strength $\hat{\sigma}$ is another important interface parameter. The physical meaning of these parameters can be displayed by a cohesive zone (CZ) model. Figure 1 illustrates the configuration of a peel test with film thickness $t$, peel force $P$ and peel angle $\Phi$. The peeling process along the interface between the film and the substrate is described by the $\mathrm{CZ}$ model, as 


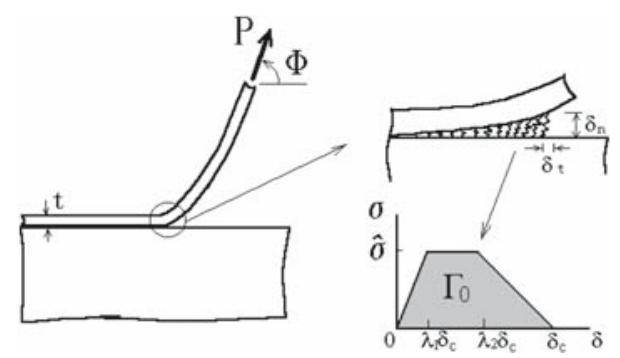

Fig. 1 Peel test configuration and sketch of cohesive zone model

shown in the right part of Fig. 1. The CZ model includes two important parameters, $\Gamma_{0}$ and $\hat{\sigma}$, (Pardoen etal. 2005; Wei 2002, 2004; Yang and Thouless 2001; Yang et al. 1999, 2001; Ferracin et al. 2003; Wei and Hutchinson 1998). In the peel test, the steady-state peel force $P$ (called the energy release rate) can be recorded, and this is enough to measure $\Gamma_{0}$ for an elastic peeling process. However, for an elastic-plastic peeling problem, due to plastic dissipation, the required energy release rate $P(1-\cos \Phi)$ to realize a steady-state peeling state is mainly contributed by not only the interface fracture energy (toughness) $\Gamma_{0}$, but also the plastically dissipated energy $\Gamma_{\mathrm{P}}$. So at steady-state delamination of the thin film, we have Eq. 1 from energy balance

$P(1-\cos \Phi)=\Gamma_{0}+\Gamma_{\mathrm{P}}$

In most cases $\Gamma_{\mathrm{P}}$ makes a large contribution to the energy release rate $P(1-\cos \Phi)$. So an appropriate procedure is needed to derive $\Gamma_{0}$ from peel test dada (Cotterell etal. 2006; Pardoen etal. 2005; Yang and Thouless 2001; Yang etal. 1999, 2001; Dillard and Pocius 2002; Kim and Aravas 1988; Kinloch et al. 1994).

In order to determine two interfacial parameters, in addition to measuring the peel force, we need to measure another quantity in the experiment. If we take both the steady-state peel force and the film curvature radius at the crack tip as the quantities to be measured, combining with the peel test theoretical analyses (Wei 2004; Wei and Hutchinson 1998; Kim and Aravas 1988; Kinloch et al. 1994; Kim et al. 1989), the interface parameters $\left(\Gamma_{0}, \hat{\sigma}\right)$ can be determined. There are two kinds of analytical methods for peel tests of elastic-plastic thin films: the beam bending model introduced by Kim et al. (Kim and Aravas 1988; Kim et al. 1989) and the rigorous plane strain finite element model presented by Wei and Hutchinson (Wei 2002, 2004; Wei and Hutchinson 1998). After comparing the two model results with experimental measurements, Wei et al. (Wei et al. 2006) concluded that the beam bending model was suitable only for a thick film case (i.e., a case with a large fracture process zone size), while the plane-strain finite element model worked well in both the thick film case and thin film case (i.e., both the large scale and small scale cases of the fracture process zone). A length scale feature in the film peeling problems can be described through the magnitude of a normalized parameter $t / R_{0}$, where $t$ is the film thickness and

$R_{0}=\left(\hat{\sigma} / \sigma_{Y}\right)\left(E / \sigma_{Y}\right) \delta_{c} /\left[3 \pi\left(1-v^{2}\right)\right]$

is the plastic zone size near the tip under the small scale yielding. The critical separation displacement $\delta_{c}$ is another important parameter in the $\mathrm{CZ}$ model; it can be expressed in terms of the two independent parameters, $\left(\Gamma_{0}, \hat{\sigma}\right)$ (see Fig. 1). $E$, $v$, and $\sigma_{Y}$ are the Young's modulus, Poisson's ratio, and yield strength of the film, respectively. The film thicknesses considered in this paper are in the range of $1 \sim 15 \mu \mathrm{m}$. As mentioned above, in order to determine the interface parameters $\left(\Gamma_{0}, \hat{\sigma}\right)$ for the elastic-plastic film case, we need to measure both the peel force and the film curvature radius $R_{\mathrm{B}}$ at the crack tip (Wei and Hutchinson 1998; Kim and Aravas 1988; Kinloch et al. 1994; Kim etal. 1989; Wei etal. 2006; Moidu and Sinclair 1995; Moidu etal. 1998). However, from our experiments we found that an unstable peeling process occurred when the film thickness was smaller than $10 \mu \mathrm{m}$ (see Fig. 5); the film made a sharp angle at the crack tip, and delaminated with cyclic folding and unfolding. In this case bending curvature radius $R_{\mathrm{B}}$ of the film is unmeasurable. So in the present research, we shall use a film thickness of $15 \mu \mathrm{m}$ to determine the interface parameters.

In the past two decades, much research on thin film delamination has been performed. But the film thicknesses considered were never at the micron scale, e.g., Kim and Aravas (1988) (analysis, film thickness $100 \mu \mathrm{m})$, Moidu etal. (1995, 1998) (experiment and analysis, 0.12-1.25 $\mu \mathrm{m}$ ), Park and Yu (1998) (experiment, 17-170 $\mu \mathrm{m}$ ), Yang and Thouless etal. (2001), Yang et al. $(1999,2001)$ (experiment and simulation, 1$3 \mathrm{~mm}$ ), Cui et al. (experiment and simulation, 1-3 mm) and Pardoen et al. (2005) (experiment and simulation, $0.78-1.2 \mathrm{~mm}$ ), etc. Micron scale thin film/substrate systems are widely used in engineering. It is a big challenge to do the peel tests for the micron-scale film 
thickness: more accuracy in sample preparation, experiment operation and post-testing processing, etc. On the other hand, higher-precision instruments are also needed. In the peel test simulations for the micron scale film case using the finite element model, two key points will be noted as follows.

First, the constitutive relation of micron-thick film should include the size effect characterization, because conventional elastic-plastic theory can not be used (Song and Jin 2002; Park and Yu 1998; Huang etal. 2004). Fortunately, strain gradient plasticity theories have been developed (Huang et al. 2004; Fleck and Hutchinson 1993, 1997, 2001; Wei and Hutchinson 1997), with which the size effects can be characterized. In the present research, a conventional theory of mechanism-based strain gradient plasticity (CMSG) (Huang et al. 2004) will be used for $\mathrm{Cu}$ film. Finite element method based on the CMSG theory has the same frame as the conventional method, and does not include higher-order terms in node variables (Wei and Hutchinson 1997; Wei 2006).

Second, how does one derive the interface parameters $\Gamma_{0}$ and $\hat{\sigma}$ from numerical results? Since $\mathrm{Cu}$ film is sputtered and electroplated to the $\mathrm{Al}_{2} \mathrm{O}_{3}$ substrate, there is no adhesive layer between film and substrate, and the beam bending model is invalid for the ultrathin film case (Wei et al. 2006). In the present research an inverse analysis method with artificial neural network to predict $\Gamma_{0}$ and $\hat{\sigma}$ is presented. Combined with experimental results for a $15 \mu \mathrm{m}$ thick film, the interface parameters $\Gamma_{0}$ and $\hat{\sigma}$ will be determined, then the experimental results for other film thicknesses will be verified through finite element simulations based on the CMSG theory and on the predicted values of $\left(\Gamma_{0}, \hat{\sigma}\right)$.

\section{Experiments}

\subsection{Experiment procedure}

A piece of $\mathrm{Al}_{2} \mathrm{O}_{3}$ is cut to small cubic samples $(40 \times 2 \times 5 \mathrm{~mm})$. The sample surfaces are polished, washed ultrasonically, and then dried. After the samples are dried, $\mathrm{Cu}$ film will be electroplated onto them. It is difficult to electroplate $\mathrm{Cu}$ film onto the $\mathrm{Al}_{2} \mathrm{O}_{3}$ substrate directly due to the poor conductance of the $\mathrm{Al}_{2} \mathrm{O}_{3}$. So two steps including sputtering and electroplating are considered. Since the sputtering process is very slow (the maximum sputtering velocity is several nanometers of film thickness per minute), it will take

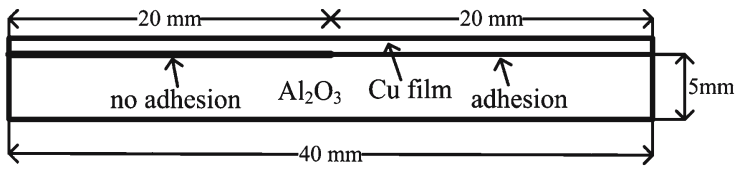

Fig. 2 Peel test sample sketch

too long time to sputter $\mathrm{Cu}$ onto the $\mathrm{Al}_{2} \mathrm{O}_{3}$ substrates with the film thickness $1 \sim 15 \mu \mathrm{m}$. Therefore, in the process of making films on the substrates, the following two steps are adopted: a $500 \mathrm{~nm}$ thick film is sputtered and then the remainder thickness of the film is electroplated. Six kinds of specimens are made which correspond to the film thicknesses, 1, 2, 3, 5, 10, and $15 \mu \mathrm{m}$, respectively. Before sputtering, some vacuum grease is coated on the left half areas of the sample surfaces on which the films will be electroplated (see Fig. 2), for making a $20 \mathrm{~mm}$ long pre-crack in each sample to facilitate the peel test operation.

All peel tests are conducted using a standard tensile testing machine with a small-scale peel test rig made specially for the current research (see Fig. 3a). A minisize sensor with high precision is used to measure the peel force. The peel angle is kept at 90 degrees and the crosshead moving velocity is kept at $0.2 \mathrm{~mm} / \mathrm{min}$ during all the peel tests. A Questar measuring microscope with long focus is used to observe the crack growth and to take micrographs. In order to keep the peel angle unchangeable during the test, a $1,000 \mathrm{~mm}$ long thin nylon thread is adopted to connect the specimen terminal to the peel test rig. Since the length of the thread is much larger than the crack propagation quantity $(20 \mathrm{~mm})$, the peel angle will be kept approximately constant during the peeling process, as shown in Fig. $3 b$.

\subsection{Experimental results}

During each peel test the peel force as a function of the crosshead displacement is recorded. Figure 4 shows two typical curves of peel force versus crosshead displacement. The initial rising part of each curve corresponds to the elongation of the detached film. Then the interface crack initiates and propagates with the increasing load. Finally the crack propagation reaches a steady-state process at a constant external load, and the active plastic zone in the film also remains the same size. For the film with the thickness $3 \mu \mathrm{m}$ the peel force reaches at a "stable" value but with a little fluctuation 
Fig. 3 Peel test rig made specifically for the current research (a)

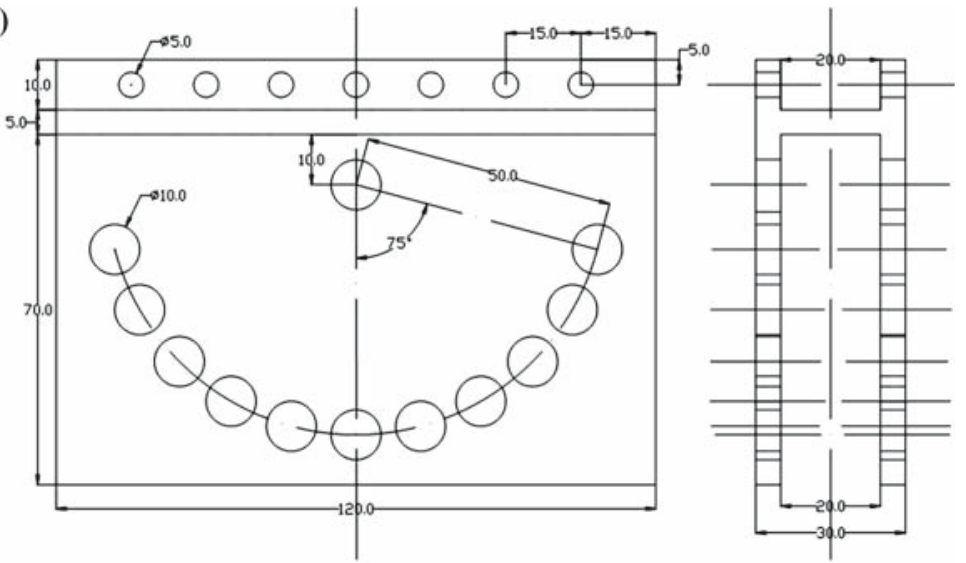

(b)

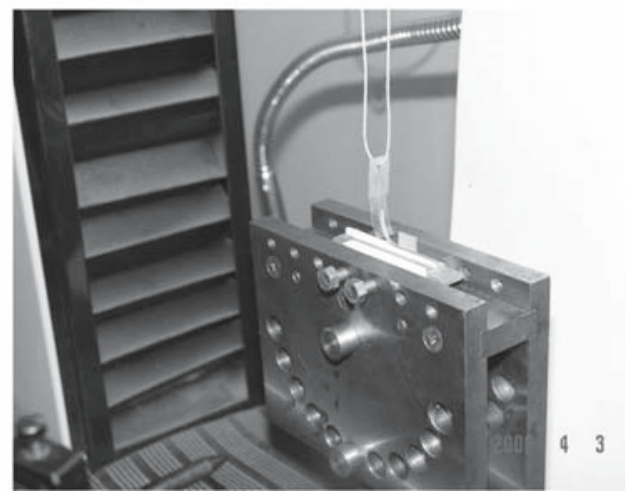

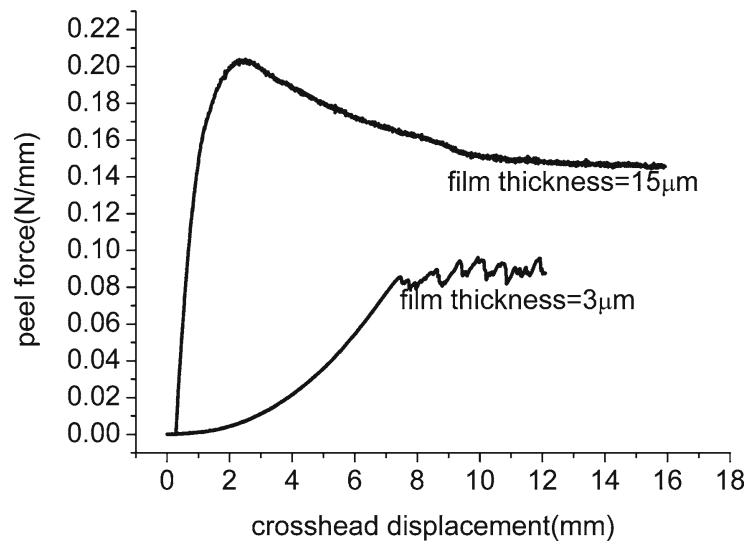

Fig. 4 Peel force variations with crosshead displacement

which corresponds to such a film delamination state at the crack tip as the film undergoes a cyclic folding and unfolding process (see Fig. 5). In this case the bending curvatures $K_{\mathrm{B}}\left(=1 / R_{\mathrm{B}}\right)$ of the film is not available.

For each case of film thickness, at least three samples are used to do the peel tests, and the mean value of the measured steady-state peel forces is plotted as a function of the film thickness, as shown in Fig. 6.

For the case of thick film, such as film thicknesses larger than $10 \mu \mathrm{m}$, the cyclic folding and unfolding phenomenon does not occur, and the bending curvature radius $R_{\mathrm{B}}\left(=1 / K_{\mathrm{B}}\right)$ of the film at the crack tip keeps constant as the peel force reaches a stable value (without fluctuation, see Fig. 4). Each value of $R_{\mathrm{B}}$ is measured using multiple points to fit the configuration of the film at the crack tip on the micrograph taken by the Questar measuring system. For the film thickness $15 \mu \mathrm{m}, R_{\mathrm{B}}=100 \mu \mathrm{m}$. This result will be used in the following Sect. 3.3 to identify the $\Gamma_{0}$ and $\hat{\sigma}$.

\section{FE simulations and neural network inverse analysis}

As pointed out in the introduction, due to considering the size effect in the peel tests with micron scale film thickness in the present investigation, mechanical behavior of the $\mathrm{Cu}$ film will be described by the CMSG 
Fig. 5 Configuration of delaminated film in the peel test when the film thickness is less than $10 \mu \mathrm{m}$

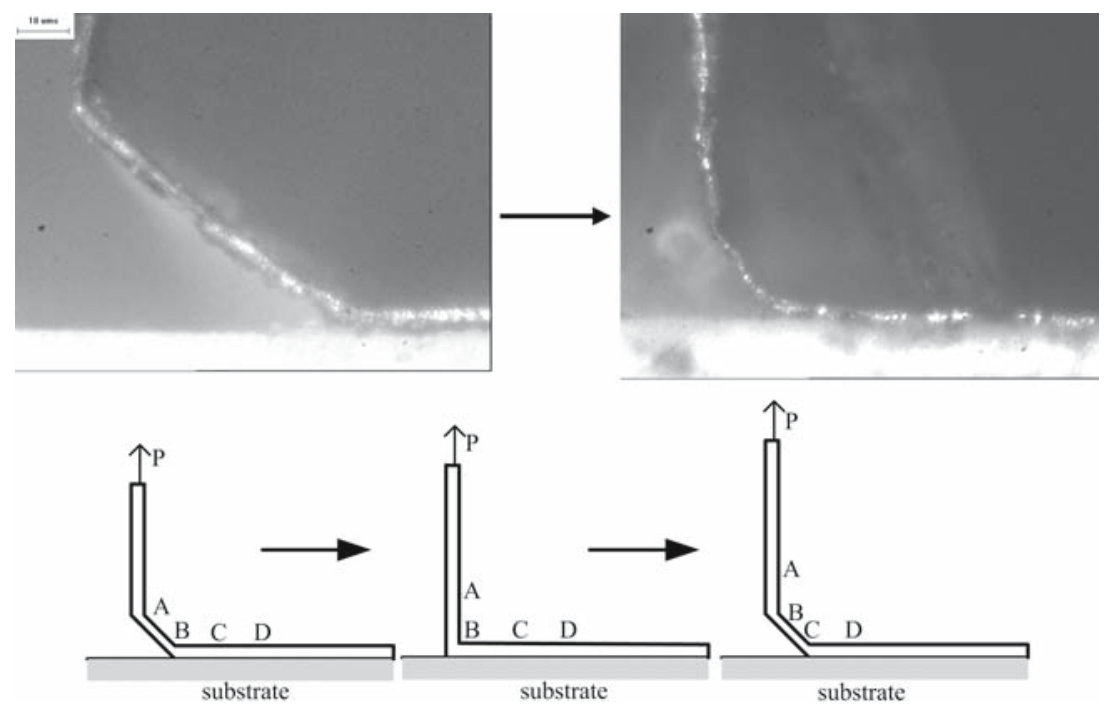

3.1 CMSG theory

Strain gradient plasticity theories are often used to describe the size effect of metallic materials at micron and sub-micron scales (Huang etal. 2004; Fleck and Hutchinson 1993, 1997, 2001; Wei and Hutchinson 1997; Wei 2006; Shi et al. 2001; Wei and Xu 2005; Wei et al. 2004). The CMSG theory does not require higherorder governing equations and additional boundary conditions. The strain gradient effect appears only in the constitutive relation as a modifying quantity in the plastic hardening modulus (Huang et al. 2004),

$$
\begin{aligned}
\dot{\sigma}_{i j}= & K \dot{\varepsilon}_{k k} \delta_{i j} \\
& +2 \mu\left\{\dot{\varepsilon}_{i j}^{\prime}-\frac{3 \dot{\varepsilon}}{2 \sigma_{e}}\left(\frac{\sigma_{e}}{\sigma_{Y} \sqrt{f^{2}\left(\varepsilon^{p}\right)+l \eta^{p}}}\right)^{m} \sigma_{i j}^{\prime}\right\}
\end{aligned}
$$

theory in our finite element simulations. For comparison, we also adopt the conventional plasticity theory to carry out the simulations. The CMSG theory is briefly introduced in the following Sect. 3.1. In order to clearly describe the interfacial peeling process, the $\mathrm{CZ}$ model is used. Foundation of the peel test finite element model is presented in the following Sect. 3.2. The determination of the interfacial parameters, $\Gamma_{0}$ and $\hat{\sigma}$, from experimental results using a two layer back propagation neural network is given in the following Sect. 3.3. At last in Sect. 3.4, a validation of the identified $\Gamma_{0}$ and $\hat{\sigma}$ is made by comparing the results of the finite element simulations and the experimental results. where $K=E /(3-6 v)$ is bulk modulus, $\mu$ is shear modulus, $\sigma_{Y}$ is yield strength, $\sigma_{e}=\sqrt{3 / 2 \sigma_{i j}^{\prime} \sigma_{i j}^{\prime}}$ is Mises effective stress, $\sigma_{i j}^{\prime}=\sigma_{i j}-1 / 3 \sigma_{k k} \delta_{i j}$ is stress deviators, $\varepsilon^{p}$ is effective plastic strain, $\dot{\varepsilon}$ is effective strain rate, $m$ is rate-sensitivity exponent of the material which takes a large value $(\geq 20)$ when rate-sensitivity is not obvious, $l$ is an intrinsic material length parameter introduced in the strain gradient plasticity theory,

$$
l=M^{2} \bar{r} \alpha^{2} b\left(\mu / \sigma_{Y}\right)^{2} \approx 18 \alpha^{2} b\left(\mu / \sigma_{Y}\right)^{2}
$$


For conventional metals $l$ is on the order of one micron. In Eq. $4 \alpha$ is an empirical coefficient about $0.3 ; b$ is the magnitude of the Burger's vector; $f\left(\varepsilon^{p}\right)$ is the ratio of elastic-plastic hardening modulus,

$f\left(\varepsilon^{p}\right)=\left(1+\frac{E \varepsilon^{p}}{\sigma_{Y}}\right)^{N}$

where $N$ is strain hardening exponent of the material. In Eq. $3 \eta^{p}$ is the effective plastic strain gradient,

$\eta^{p}=\sqrt{\frac{1}{4} \eta_{i j k}^{p} \eta_{i j k}^{p}}$

where $\eta_{i j k}^{p}=\varepsilon_{i k, j}^{p}+\varepsilon_{i k, i}^{p}-\varepsilon_{i j, k}^{p}$ is the component of plastic strain gradient tensor and $\varepsilon_{i j}^{p}=\int \dot{\varepsilon}_{i j}^{p} d t$ is the component of plastic strain tensor.

Since the effective plastic strain gradient (see Eq. 6) is usually calculated based on the result of $\varepsilon_{i j}^{p}$ at the last step, the finite element method for the CMSG model is the same as for the conventional plasticity theory. The constitutive relation of the CMSG (see Eq. 3) will degenerate into the conventional plasticity theory when $m$ tends to zero.

\subsection{Finite element model}

In the present peel tests, since the width of the films ( $2 \mathrm{~mm}$ ) is much larger than their thicknesses $(1 \sim 15 \mu \mathrm{m})$, the peel tests can be treated as plane strain problems. The finite element model using ABAQUS version 6.5 will be used.

A UMAT subroutine of ABAQUS is defined using Fortran 77 to describe the CMSG constitutive relation with large deformations, the Mises yield criterion and isotropic strain-hardening material model (ABAQUS 2004). The primary parameters of the $\mathrm{Cu}$ film are shear modulus $G=48,300 \mathrm{MPa}$, Poisson's ration $v=0.343$, initial yield stress $\sigma_{\mathrm{Y}}=70 \mathrm{MPa}$, power law hardening coefficient $N=0.32$, rate-sensitivity measuring parameter $m=20, \alpha=0.33$ and the magnitude of Burger's vector $b=0.27 \mathrm{~nm}$. The $\mathrm{Al}_{2} \mathrm{O}_{3}$ substrates undergo very small deformations during the peel tests and are treated as elastic material with Young's modulus $E=400 \mathrm{GPa}$ and Poisson's ration $v=0.3$.

The CZ model (Pardoen et al. 2005; Wei 2002, 2004; Yang and Thouless 2001; Yang etal. 1999, 2001; Dillard and Pocius 2002; Wei and Hutchinson 1998) is employed in our FE calculations to describe the interface crack traction between the film and the substrate during the peeling process (see Fig. 1). The parameters governing the traction separation law are the fracture toughness $\Gamma_{0}$, separation strength $\hat{\sigma}$, critical opening displacement ratio $\delta_{n}^{c} / \delta_{t}^{c}$ and the shape factors $\lambda_{1}$ and $\lambda_{2}$. Earlier studies show that the shape of the traction separation law is relatively unimportant (Wei 2004), so in our model we take $\lambda_{1}=0.15, \lambda_{2}=0.5$. The two most important parameters are $\Gamma_{0}$ and $\hat{\sigma}$ (Wei 2004). The parameter $\delta_{n}^{c} / \delta_{t}^{c}$ is also important in mixed mode fracture problems, but the predictions are relatively insensitive to this parameter as long as the fracture process is normal-separation dominating (Tvergaard and Hutchinson 1993), which is the case for the peel tests with a peel angle of 90 degrees considered in this paper.

When the dimensionless separation $\lambda\left(\lambda=\sqrt{\left(\delta_{\mathrm{n}} / \delta_{\mathrm{n}}^{\mathrm{c}}\right)^{2}+\left(\delta_{\mathrm{t}} / \delta_{\mathrm{t}}^{\mathrm{c}}\right)^{2}}\right.$ tends to unity at the crack tip within the $\mathrm{CZ}$, the crack grows. An intrinsic element COH2D4 in ABAQUS 6.5 can be used as the cohesive element to link the film and the substrate (ABAQUS 2004). For conveniently exerting load on the film, a rigid element is attached at the free end of the film. At first the rigid element is rotated by 90 degrees and then is displaced by the peeling force in the 90 degrees direction with a constant velocity. The film and the substrate are meshed using bi-linear rectangular elements with four nodes and four integration Gauss points. Since the film undergoes large bending deformations during the peel test, at least four element layers will be adopted along the film thickness in order to capture the deformations. Since the Young's modulus of the substrate material $\mathrm{Al}_{2} \mathrm{O}_{3}$ is about three times larger than that of the $\mathrm{Cu}$ film, the substrate deformation is much smaller than the film deformation during the peeling; therefore, sparse mesh is adopted in the substrate region in order to increase the calculation efficiency. Figure 7 shows a typical mesh used in our finite element simulations.

\subsection{Inverse analysis using a neural network to predict} $\Gamma_{0}$ and $\hat{\sigma}$

Since both the interfacial fracture energy $\Gamma_{0}$ and separation strength $\hat{\sigma}$ are the most important parameters in the fracture research of the interface (Evans etal. 1999), we select them as the target to be measured in the present research. Here an inverse analysis method 
Fig. 7 A typical mesh used in the FE calculations

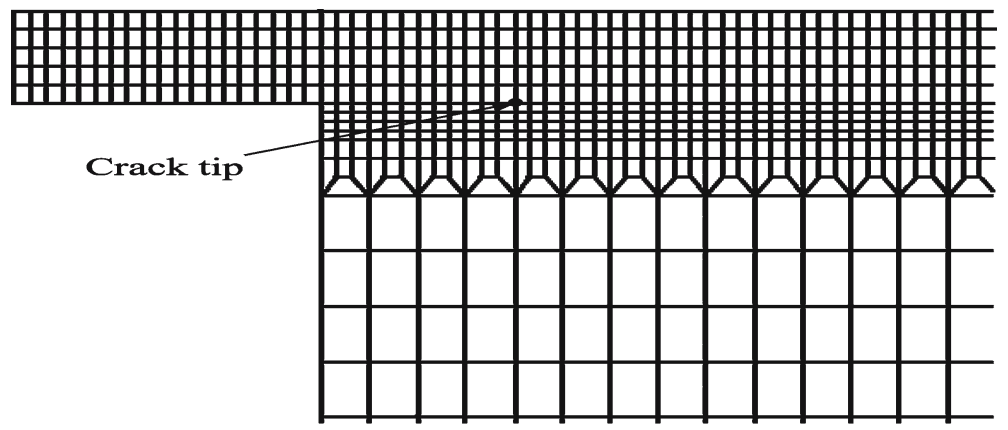

is presented to identify the parameters $\Gamma_{0}$ and $\hat{\sigma}$ using the artificial neural network method.

For the film thickness $15 \mu \mathrm{m}$, the peeling process is stable. Both the peel force $P$ and the bending curvature radius $r\left(=R_{\mathrm{B}}\right)$ of the film at the crack tip can be described uniquely by the interfacial parameters $\Gamma_{0}$ and $\hat{\sigma}$,

$$
\begin{aligned}
& P=f_{1}\left(\Gamma_{0}, \hat{\sigma}\right) \\
& r=g_{1}\left(\Gamma_{0}, \hat{\sigma}\right)
\end{aligned}
$$

We also have the inverse relations

$$
\begin{aligned}
& \Gamma_{0}=f_{2}(P, r) \\
& \hat{\sigma}=g_{2}(P, r)
\end{aligned}
$$

Both $f_{2}$ and $g_{2}$ can be determined numerically by the neural network method.

In the inverse analysis based on the neural network method, the finite element solutions are firstly used as training data to train a neural network. Given a series of values $\left(\Gamma_{0}^{i}, \hat{\sigma}^{i}\right)$, one can obtain the same number of values $\left(P^{i}, r^{i}\right)$ using the finite element method. The obtained results are used as input data for training the neural network, while the values $\left(\Gamma_{0}^{i}, \hat{\sigma}^{i}\right)$ are used as target data. From the experimental results shown in Fig. 6, one can find the region of the interfacial fracture energy $\Gamma_{0}<0.07 \mathrm{~N} / \mathrm{mm}$. So for the series $\left(\Gamma_{0}^{i}, \hat{\sigma}^{i}\right)$, we take six values of $\Gamma_{0}$ in the range $[0.01,0.06]$ and 11 values of $\hat{\sigma}$ in a large range [5, 50]. Through finite element calculations, we have 66 values of $\left(P^{i}, r^{i}\right)$. Comparing the calculated $\left(P^{i}, r^{i}\right)$ with the experimental data for the $15 \mu \mathrm{m}$ thick film $(P=0.146 \mathrm{~N} / \mathrm{mm}, r=0.10 \mathrm{~mm})$, one can find that the true values of both $\Gamma_{0}$ and $\hat{\sigma}$ do fall into the range $[0.01,0.06]$ and $[5,50]$, respectively.

A two-layer feed-forward backpropagation network with two inputs and two outputs is built in MATLAB version 7.0 (MATLAB 2004). There are seven nerve

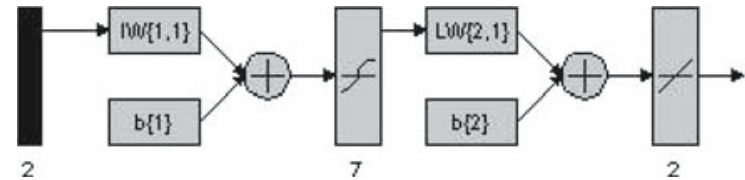

Fig. 8 Sketch of the neural network

cells in the first layer and the transfer function is TANSIG. The second layer has two nerve cells and the transfer function is PURELIN. TRAINLM is used as the training function for the whole network. A sketch of the neural network is shown in Fig. 8. This network can simulate any function with two dependent and two independent variables, providing that the function is not continuous only at finite points (MATLAB 2004).

The network described in Fig. 8 is trained by the 66 values of $\left(P^{i}, r^{i}\right)$ and $\left(\Gamma_{0}^{i}, \hat{\sigma}^{i}\right)$, noting that these values are obtained using the finite element calculations with the CMSG theory. The variations of the $g_{2}$ and $f_{2}$ based on the neural network method are shown in Figs. 9 and 10 , respectively. In these figures, $\Gamma$ and $\sigma$ stand for the values of $\left(\Gamma_{0}, \hat{\sigma}\right)$ to be determined, and $\mathrm{T}$ is the target value.

From Fig.9 and Fig.10, obviously, the simulated $f_{2}$ and $g_{2}$ by the trained neural network are considerably accurate. By inputting the experimental data $(P=0.146$ $\mathrm{N} / \mathrm{mm}, r=0.10 \mathrm{~mm}$ ) into the trained network, one can obtain, $\Gamma_{0}=0.045 \mathrm{~N} / \mathrm{mm}, \hat{\sigma}=26.6 \mathrm{MPa}$.

For the finite element calculations based on the conventional elastic-plastic theory, by repeating the above procedure one can obtain the results shown in Figs. 11 and 12. By inputting the experimental data $(P=0.146$ $\mathrm{N} / \mathrm{mm}, r=0.10 \mathrm{~mm}$ ) into the trained network, one can obtain the predicted result $\Gamma_{0}=0.047 \mathrm{~N} / \mathrm{mm}, \hat{\sigma}=24.9$ $\mathrm{MPa}$. There is small difference between the two predictions. Since the film thickness considered in the predictions is $15 \mu \mathrm{m}$, size effect is not obvious. However, if 


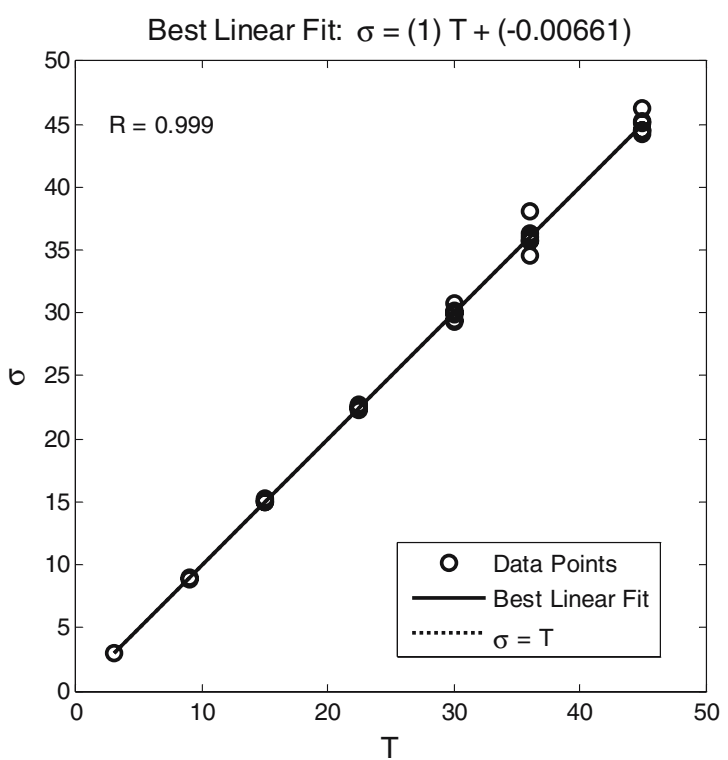

Fig. 9 Variation of $g_{2}$ based on the CMSG theory. The relation of predicted value $(\sigma)$ using the neural network with target value (T)

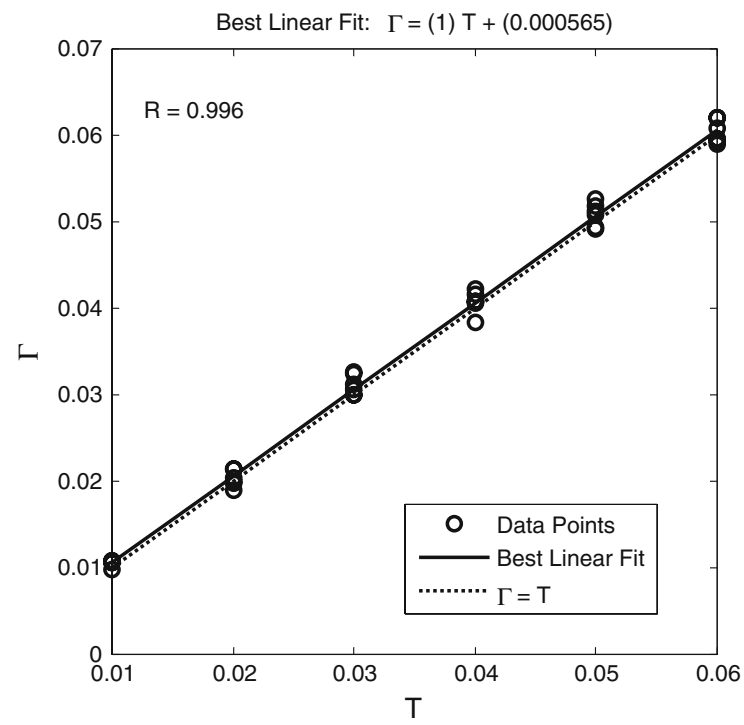

Fig. 10 Variation of $f_{2}$ based on the CMSG theory. The relation of predicted value $(\Gamma)$ using the neural network with target value (T)

we adopt the thinner film in our analysis (see the following Sect. 3.4), the two predicted results show some considerable difference.

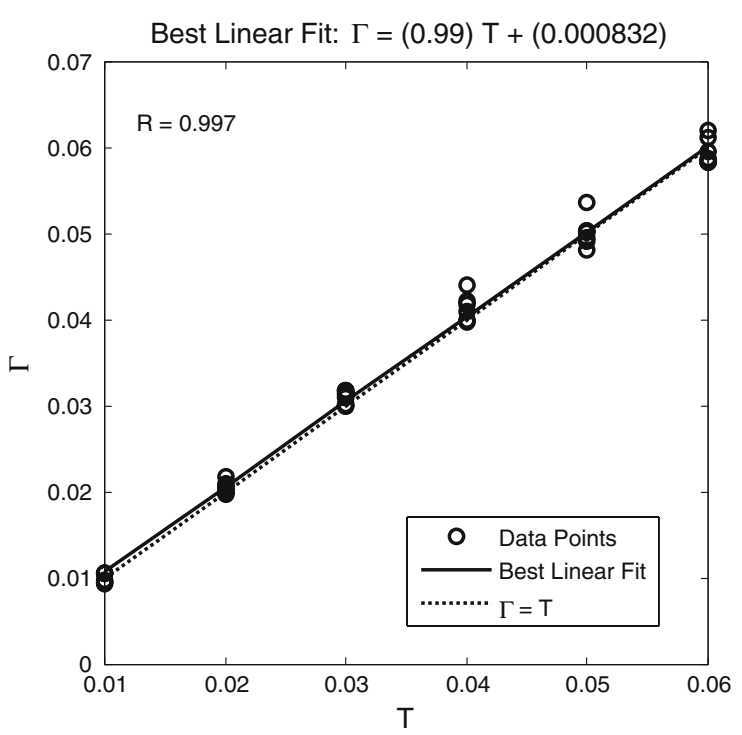

Fig. 11 Variation of $f_{2}$ based on the conventional theory. The relation of predicted value $(\Gamma)$ using the neural network with target value $(\mathrm{T})$

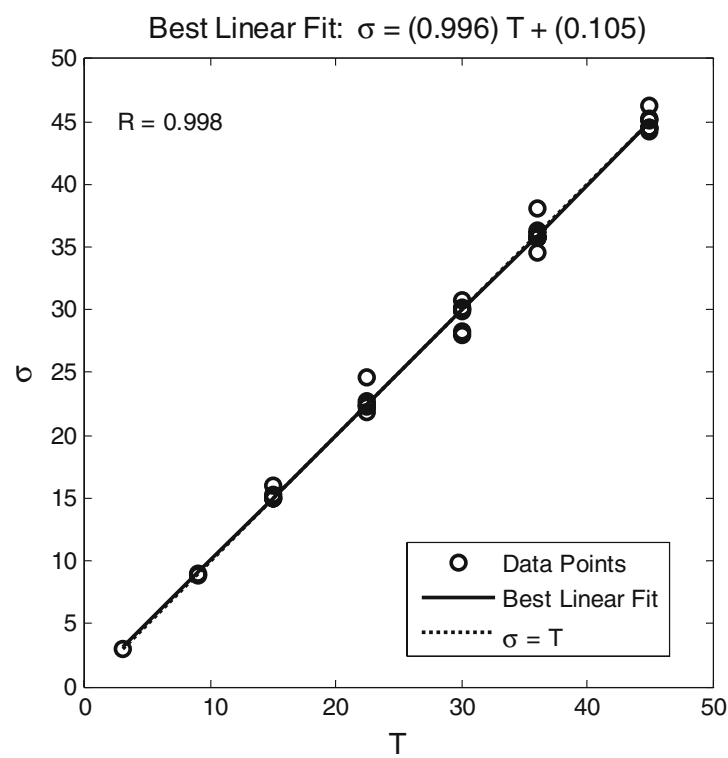

Fig. 12 Variation of $g_{2}$ based on the conventional theory. The relation of predicted value $(\sigma)$ using the neural network with target value $(\mathrm{T})$

\subsection{Validation of the predictions made by the neural network}

In order to validate the predictions by the neural network, we consider six film thicknesses ranging from 1 to $15 \mu \mathrm{m}$. The predicted results are then compared 


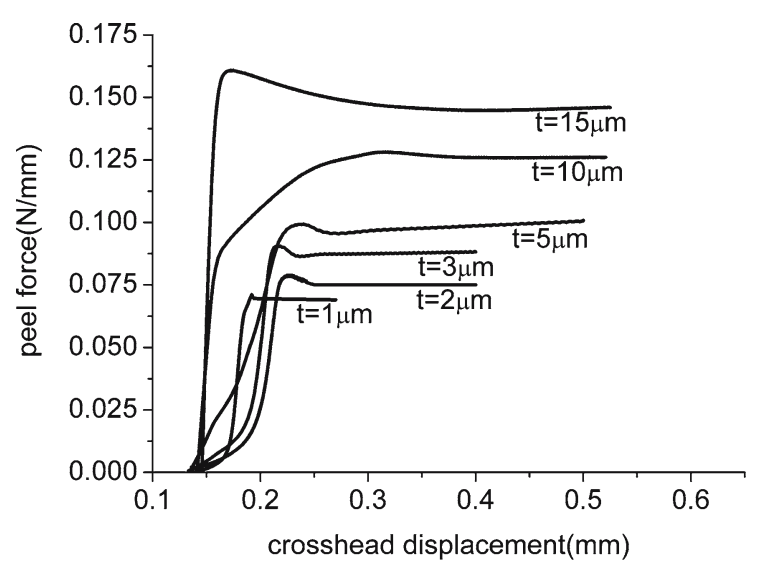

Fig. 13 Simulated peel force as a function of crosshead displacement for different film thickness based on the predicted results $\Gamma_{0}=0.045 \mathrm{~N} / \mathrm{mm}, \hat{\sigma}=26.6 \mathrm{MPa}$

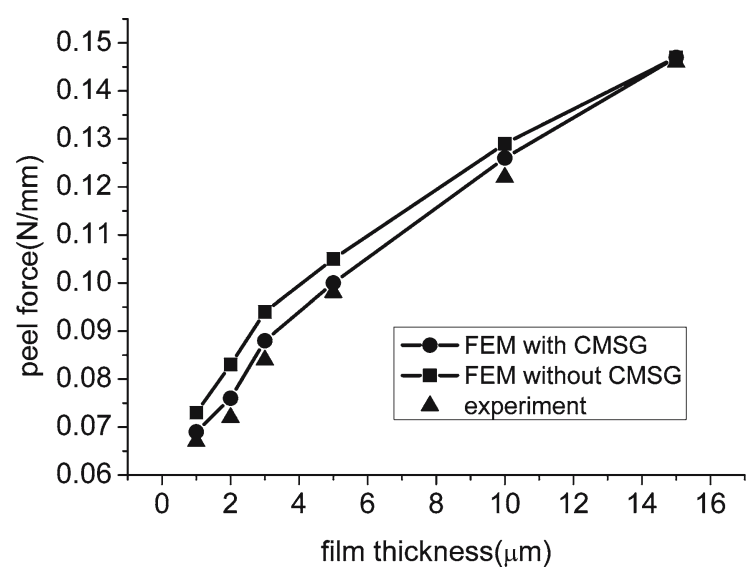

Fig. 14 Steady-state peel force as a function of film thickness

with the experimental results. The variations of peel force vs. crosshead displacement for the six film thicknesses using the finite element calculations based on the CMSG theory are shown in Fig. 13. The trends of the curves (peel force vs. displacement; peel force vs. film thickness) in Fig. 13 are similar to those of the experimental curves shown in Figs. 4 and 6.

Figure 14 shows the variations of the peel forces measured in the experiments and simulated by the finite element models with and without considering the strain gradient effect. From Fig. 14, the simulated peel forces using the CMSG finite element model with the predicted parameters $\left(\Gamma_{0}=0.045 \mathrm{~N} / \mathrm{mm}, \hat{\sigma}=26.6 \mathrm{MPa}\right)$ agree well with the experimental results. The finite element model without CMSG (the conventional plasticity theory) gives larger peel force predictions than the experimental results. The conventional plasticity theory overestimates the plastic dissipation energy.

\section{Concluding Remarks}

Peel test measurements have been performed to estimate the interface toughness and separation strength between thin copper film with micron scale thickness and $\mathrm{Al}_{2} \mathrm{O}_{3}$ substrate. In the present research, the film thicknesses considered range from 1 to $15 \mu \mathrm{m}$. The measured peel forces reach at the stable values (corresponding to steady-state peeling) but with a slight fluctuation when the film thickness is smaller than $10 \mu \mathrm{m}$. From the experimental observation, for the case of very thin film, the slight fluctuation of peel force corresponds to the film peeling process at the crack tip like that the film undergoes cyclic folding and unfolding deformation during the peeling process. Through measuring the steady-state peel force and the film curvature radius, the important interface parameters $\left(\Gamma_{0}\right.$ interfacial fracture energy; $\hat{\sigma}$ interfacial separation strength) have been derived out using the artificial neural network method. In the inverse analyses, in order to investigate the size effect in the peeling of micron-scale film, the finite element results based on both the CMSG strain gradient theory and the conventional elastic-plastic theory have been adopted respectively to train the neural network. Using the two kinds of theories, we obtain $\left(\Gamma_{0}=0.045 \mathrm{~N} / \mathrm{mm}, \hat{\sigma}=26.6 \mathrm{MPa}\right)$ and $\left(\Gamma_{0}=0.047 \mathrm{~N} / \mathrm{mm}\right.$, $\hat{\sigma}=24.9 \mathrm{MPa}$ ), based on the CMSG theory and on the conventional elastic-plastic theory, respectively. We find that there is small difference between the two predictions. This is because the film thickness adopted in the two predictions is $15 \mu \mathrm{m}$, in which case the film is so thick that the size effect is not obvious. However, when we predict the peel tests with the thinner films based on the above parameter values, the obtained two peel forces display considerable difference.

Note that in the present research, the interface parameters have been determined using the experimental data for the film thickness 15 microns (corresponding to having a measurable curvature radius at the crack tip during the steady-state peeling). However, when the film thickness is smaller than $10 \mu \mathrm{m}$, there is no measurable curvature radius at the crack tip (see Fig. 5), in which case the present scheme is invalid, and one needs to explore other methods to determine the interface parameters. On the other hand, from the present re- 
search, the size effect is significant when the film thickness is smaller than $10 \mu \mathrm{m}$ (see Fig. 14). One can not adopt the conventional elastic-plastic finite element solutions to train the neural network in the inverse analysis in order to obtain the reasonable prediction of the interface parameters. Certainly, one can use some scaling theories (like the strain gradient plasticity theories) to train the neural network, however this is also limited when the film thickness is smaller than submicron.

Acknowledgements This work is supported partially by the Chinese Academy of Sciences and partially by National Science foundation of China through Grants 10432050, 10428207, and 10672163.

\section{References}

ABAQUS Inc (2004) ABAQUS Version 6.5 Documentation

Asai H, Iwase N, Suga T (2001) Influence of ceramic surface treatment on peel-off strength between aluminum nitride and epoxy-modified polyaminobismaleimide adhesive. IEEE Trans Adv Pack 24: 104-112

Bundy K, Schlegel U, Rahn B et al (2000) Improved peel test method for measurement of adhesion to biomaterials. J Mater Sci: Mater in Med 11: 517-521

Cotterell B, Hbaieb K, Williams JG et al (2006) The root rotation in double cantilever beam and peel tests. Mech Mater 38: $571-584$

Cui J, Wang R, Sinclair AN et al (2003) A calibrated finite element model of adhesive peeling. Int J Adhes Adhesives 23: 199-206

Dillard DA, Pocius AV (eds) (2002) The mechanics of adhesion. Elsevier press

Evans AG, Hutchinson JW, Wei Y (1999) Interface adhesion: effects of plasticity and segregation. Acta Mater 47: 40934113

Ferracin T, Landis CM, Delannay F et al (2003) On the determination of the cohesive zone properties of an adhesive layer from the analysis of the wedge-peel test. Int J Solids Struct 40: 2889-2904

Fleck NA, Hutchinson JW (1993) A phenomenological theory for strain gradient effects in plasticity. J Mech Phys Solids 41: $1825-1857$

Fleck NA, Hutchinson JW (1997) Strain gradient plasticity. Adv Appl Mech 33: 295-361

Fleck NA, Hutchinson JW (2001) A reformulation of strain gradient plasticity. J Mech Phys Solids 49: 2245-2271

Hadavinia H, Kawashita L, Kinloch AJ et al (2006) A numerical analysis of the elastic-plastic peel test. Eng Fract Mech 73: 2324-2335

Huang Y, Qu S, Hwang KC et al (2004) A conventional theory of mechanism-based strain gradient plasticity. Int J Plast 20: 753-782

Kim KS, Aravas N (1988) Elasto-plastic analysis of the peel test. Int J Solids Struct 24: 417-435

Kim J, Kim KS, Kim YH (1989) Mechanical effects of peel adhesion test. J Adhes Sci Tech 3: 175-187
Kinloch AJ, Lau CC, Williams JG (1994) The peeling of flexible laminates. Int J Fract 66: 45-70

MATLAB Inc (2004) MATLAB Version 7.0 Documentation

Moidu AK, Sinclair AN, Spelt JK (1995) Analysis of the peel test: prediction of adherend plastic dissipation and extraction of fracture energy in metal-to-metal adhesive joints. $\mathrm{J}$ Test Evaluat 23: 241-253

Moidu AK, Sinclair AN, Spelt JK (1998) On the determination of fracture energy using the peel test. J Test Evaluat 26: $247-254$

Pardoen T, Ferracin T, Landis CM et al (2005) Constraints effects in adhesive joint fracture. J Mech Phys Solids 53: 1951-1983

Park IS, Yu J (1998) An X-ray study on the mechanical effects of the peel test in a $\mathrm{Cu} / \mathrm{Cr} /$ polyimide system. Acta mater 46 : 2947-2953

Park YB, Park IS, Yu J (1999) Interfacial fracture energy measurement in the $\mathrm{Cu} / \mathrm{Cr} /$ polyimide system. Mater Sci Eng A 266: 261-266

Shi M, Huang Y, Jiang H et al (2001) The boundary-layer effect on the crack tip field in mechanism-based strain gradient plasticity. Int J Fract 112: 23-41

Song JY, Yu J (2002) Analysis of the T-peel strength in a $\mathrm{Cu} / \mathrm{Cr} /$ Polyimide system. Acta Mater 50: 3985-3994

Tvergaard V, Hutchinson JW (1993) The influence of plasticity on mixed mode interface fracture. J Mech Phys Solids 41: $1119-1135$

Wei Y (2002) Thin layer splitting along the elastic-plastic solid surface. Int J Fract 113: 233-252

Wei Y (2004) Modeling nonlinear peeling of ductile thin filmscritical assessment of analytical bending models using FE simulations. Int J Solids Struct 41: 5087-5104

Wei Y (2006) A new finite element method for strain gradient theories and applications to fracture analyses. Eur J Mech A/Solids 25: 897-913

Wei Y, Hutchinson JW (1997) Steady-state crack growth and work of fracture for solids characterized by strain gradient plasticity. J Mech Phys Solids 45: 1253-1273

Wei Y, Hutchinson JW (1998) Interface strength, work of adhesion and plasticity in the peel test. Int J Fract 93: 315-333

Wei Y, Xu G (2005) A multiscale model for the ductile fracture of crystalline materials. Int J Plast 21: 2123-2149

Wei Y, Qiu X, Hwang KC (2004) Steady-state crack growth and fracture work based on the theory of mechanism-based strain gradient plasticity. Eng Fract Mech 71: 107-125

Wei Y, Zhao H, Cao A (2006) Modeling and measurement of plastic dissipation in micron-thickness thin film peeling, The 12th International Symposium on Plasticity, Halifax, Canada, July 17-22

Yang QD, Thouless MD (2001) Mixed-mode fracture analyses of plastically-deforming adhesive joints. Int J Fract 110: 175-187

Yang QD, Thouless MD, Ward SM (1999) Numerical simulations of adhesively-bonded beams failing with extensive plastic deformation. J Mech Phys Solids 47: 1337-1353

Yang QD, Thouless MD, Ward SM (2001) Elastic-plastic modeII fracture of adhesive joints. Int J Solids Struct 38: 32513262 